\title{
Doença de Crohn leve a moderada
}

Adérson Omar Mourão Cintra Damião

A doença de Crohn (DC) representa um dos fenótipos das "doenças inflamatórias intestinais" (DII), juntamente com a retocolite ulcerativa (RU) e a colite não classificada (CNC). Sua fisiopatologia envolve diversas mutações genéticas nas vias de regulação da imunidade intestinal, as quais determinam uma reatividade inflamatória aumentada à microbiota com possível interferência de fatores ambientais ainda não bem esclarecidos. Como resultado, desenvolve-se um processo inflamatório crônico e inespecífico que se inicia no nível da mucosa com posterior envolvimento de camadas mais profundas, como a muscular própria e a serosa (inflamação transmural ${ }^{1,2}$. A DC pode ser classificada em subtipos correspondentes a idade, localização anatômica e comportamento (classificação de Montreal - Quadro 1). A agressividade da DC permite, por outro lado, classificá-la em diferentes graus de atividade inflamatória (ver a seguir). Essas informações podem ser determinantes quanto às condutas adotadas no decorrer da evolução da doença $a^{3,4}$.

Muitas classificações foram propostas para a DC ao longo dos anos. A mais utilizada e aceita na atualidade é a classificação de Montreal', a qual se baseia em três parâmetros principais: idade ao diagnóstico, localização e comportamento da doença. Por causa dos termos em inglês que deram origem aos três itens, a caracterização é feita com as letras A (age), L (location) e B (behavior). 


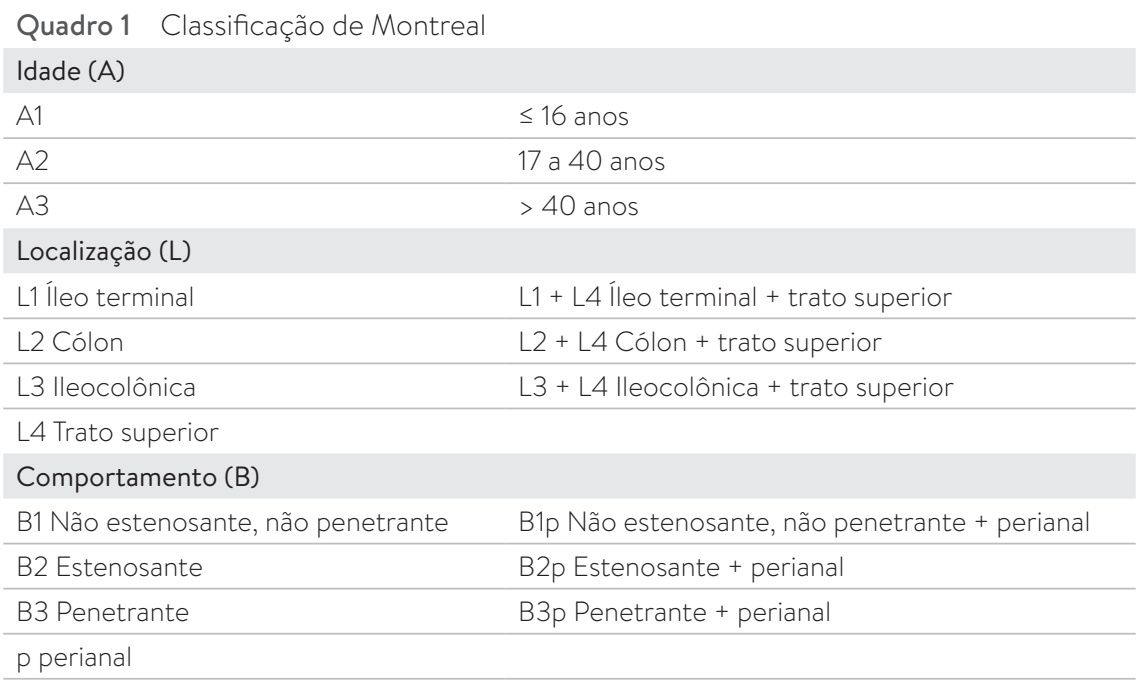

As diferentes características apontadas guardam uma relação com prognóstico, evolução para complicações e orientação terapêutica ${ }^{5}$.

A remissão é habitualmente definida do ponto de vista clínico, considerando o controle dos sintomas em um determinado momento, podendo ser induzida pelo tratamento ou espontânea. Entretanto, quando o controle dos sintomas estiver vinculado ao uso de corticosteroides (recidiva ocorrendo no desmame), o paciente não deve ser considerado "em remissão", mas, sim, "dependente de corticosteroide" 4 . Existe atualmente uma tendência para que o conceito de remissão na DII se estenda além da observação clínica e seja extrapolado para o controle paralelo dos marcadores inflamatórios laboratoriais (calprotectina fecal, proteína C-reativa) e dos achados de imagem (colonoscopia, enterografia), também chamada de "remissão profunda". Esse novo conceito ressalta a necessidade do controle objetivo da inflamação, e não simplesmente da sintomatologia, a fim de que sejam evitadas complicações de longo prazo pela persistência do processo inflamatório em pacientes com inflamação subclínica, possivelmente modificando a história natural da DC e evitando a evolução para complicações como estenoses e fístulas ${ }^{6}$.

A doença ativa, por outro lado, pode ser classificada quanto ao grau do processo inflamatório em leve, moderada, grave e "fulminante". Esta "quantificação" da inflamação pode ser feita de forma menos objetiva a partir da avaliação clínica ou calculada com índices de atividade como o Crohn's Disease Activity Index (CDAI) ou Harvey-Bradshaw Index (HBI), estes habitualmente usados em pesquisa. Os índices de atividade consideram não somente a sintomato- 
logia diretamente ligada às manifestações inflamatórias intestinais, mas também às manifestações extraintestinais. A remissão clínica definida pelo CDAI equivale a uma pontuação $<150$. A percepção clínica do paciente em atividade leve a moderada (CDAI 150 até 220) é aquela do indivíduo sintomático ambulatorial, capaz de tolerar alimentação oral, sem sinais de desidratação, obstrução intestinal, toxicidade sistêmica (febre, calafrios, prostração), massa ou defesa abdominais e que não perderam mais de $10 \%$ do seu peso. Os pacientes com atividade moderada a grave (CDAI entre 220 e 450) podem apresentar falha ao tratamento inicial, além de febre, perda ponderal significativa, dor importante e/ou defesa abdominais, náuseas, vômitos, distensão abdominal e anemia. Aqueles com atividade fulminante (CDAI > 450) geralmente evoluem com falha a todos os medicamentos disponíveis (corticosteroides venosos, imunossupressores e medicação biológica), prostração intensa, incapacidade de ingerir alimentos, sinais de obstrução intestinal, reação peritoneal, febre elevada, caquexia ou evidência de abscessos abdominais ${ }^{4}$. O conceito de doença localizada se aplica a um envolvimento $<30 \mathrm{~cm}$ do trato digestivo, enquanto a doença extensa se aplica a um envolvimento $>100 \mathrm{~cm}$. A resposta a qualquer tratamento na DC é geralmente definida como uma redução do CDAI $\geq 100$ pontos em relação ao índice inicial ${ }^{3}$.

É importante salientar que, ao longo de muitos anos e até o presente, as escolhas terapêuticas na DC têm sido fundamentadas em grande parte no grau de atividade do processo inflamatório e na sua localização. No entanto, publicações mais atualizadas chamam atenção para a presença de determinados fatores prognósticos de gravidade em alguns pacientes, muitas vezes revelados desde o início dos sintomas e que não necessariamente elevam o "grau de atividade" calculado pelos índices clássicos. Na DC, temos como exemplos: idade jovem ao diagnóstico, necessidade de corticoterapia precoce, envolvimento do trato digestivo superior, doença perianal, ulcerações profundas, tabagismo, reatividade sorológica e presença de mutação NOD2. Portanto, a correta identificação de maus fatores prognósticos pode também ser decisiva quanto à escolha terapêutica, apesar de se estar diante de uma doença momentaneamente "leve" ou "moderada", porém contendo elementos de possível evolução grave no quadro clínico ${ }^{3,7}$.

O tratamento inicial da DC e as mudanças terapêuticas ao longo do acompanhamento devem ser sempre pautadas na identificação objetiva da inflamação a fim de que sejam evitados erros de interpretação dos sintomas por outras causas frequentes como superposição com queixas funcionais ou decorrentes de complicações como abscessos, fístulas, estenoses, infecções, supercrescimento bacteriano e má absorção de sais biliares. A budesonida oral é o medicamento de escolha para indução da remissão na DC leve restrita à região ileo- 
cecal. Segundo algumas publicações, até um terço dos pacientes com DC pode ter uma evolução benigna sem complicações ao longo de muitos anos, não necessitando de terapia imunossupressora sistêmica, seja com imunossupressores (azatioprina/6-mercaptopurina, metotrexato) ou com medicação biológica. Isso justificaria uma tentativa inicial com a budesonida, nestes casos de doença localizada da região ileocecal, pela possibilidade de alguns pacientes atingirem remissão prolongada em formas leves da doença após um curso breve de corticosteroide. A ação clínica da budesonida nesse contexto é comparativamente inferior ao uso de corticosteroides sistêmicos, como prednisona/prednisolona, porém com menos efeitos colaterais hormonais. Espera-se que 50 a $60 \%$ desses pacientes entrem em remissão clínica com budesonida na dose diária de $9 \mathrm{mg}$, via oral, em um período de 8 semanas $^{3,8}$. Assim como outros corticosteroides, a budesonida não está indicada como tratamento de manutenção da remissão na DC, uma vez que não há evidência clara de benefício clínico na literatura médica sobre seu uso além de um período de 3 meses ${ }^{9}$.

Os pacientes com DC ileocecal de grau moderado podem ser tratados com budesonida, prednisona ou prednisolona. O uso de corticosteroides de ação sistêmica deve seguir recomendações de dose, tempo de uso e desmame para que sejam minimizados seus efeitos colaterais hormonais. Eles não devem ser usados para manter remissão. Aceita-se em geral que a necessidade de mais de um curso de corticosteroide por ano já represente um gatilho para o escalonamento terapêutico com uma droga que consiga manter remissão livre de corticosteroides como imunossupressores (AZA/6-MP e metotrexato). A falha a imunossupressores determinaria habitualmente a indicação da medicação biológica, a qual pode ser realizada com drogas anti-TNF (infliximabe, adalimumabe e certolizumabe) ou anti-integrina (vedolizumabe). Naturalmente, os pacientes com atividade inflamatória refratária ao uso de corticosteroides ou aqueles que apresentem intolerância aos seus efeitos colaterais hormonais poderão ter sua conduta modificada mais precocemente para imunossupressores e/ou biológicos, prescritos em associação ou isoladamente a depender do contexto clínico e do tipo de biológico indicado ${ }^{3}$.

A DC colônica leve a moderada pode ser tratada com corticoterapia sistêmica quando clinicamente relevante. As mesmas medidas descritas sobre a utilização de imunossupressores e agentes biológicos devem ser instituídas para os casos de dependência, intolerância ou refratariedade ao uso de corticosteroides ${ }^{3}$.

A DC extensa do intestino delgado deve ser interpretada como fator de risco para evolução grave e deve ser tratada com corticoterapia sistêmica, mas a introdução precoce de imunossupressores e/ou terapia biológica deve ser considerada em casos de atividade moderada ou dependentes de corticosteroi- 
des. Os casos leves de DC esofágica e/ou gastroduodenal podem se beneficiar apenas com inibidores de bomba protônica. Casos refratários devem obedecer a sequência de indicações de corticosteroides sistêmicos, imunossupressores e biológicos conforme já mencionado em outras situações.

A mesalazina tem efeito marginal na DC quando comparada a placebo, mesmo quando o grau de atividade é leve a moderado. Em uma metanálise que avaliou indução da remissão publicada em 2004, doses de 4 g/dia de mesalazina foram associadas a uma queda de 18 pontos no CDAI (variação de pontos com valor clínico questionável) para que o cálculo estatístico atingisse significância $(\mathrm{p}=0,04)^{10}$. Uma publicação em 1979 mostrou algum benefício da sulfassalazina 3 a $6 \mathrm{~g} /$ dia na indução da remissão da DC do cólon (baixo nível de evidência), mas não da DC do intestino delgado, sem benefício na manutenção da remissão ${ }^{11}$. Alguns especialistas ainda recomendam a mesalazina tópica como coadjuvante no tratamento da proctossigmoidite da $\mathrm{DC}^{3}$. Uma importante revisão sistemática mais recente realizada por um grupo de autores do American College of Gastroenterology (ACG IBD Task Force) concluiu, com base na precariedade das evidências em literatura, que não há dados científicos suficientes para se indicar aminossalicilatos para indução ou para manutenção da remissão no tratamento da DC, seja do intestino delgado, da região ileocecal ou do cólon ${ }^{12}$.

Antibióticos de ação sistêmica como metronidazol e ciprofloxacina não estão indicados para indução ou manutenção da remissão na DC. Entretanto, o antibiótico não absorvível de ação luminal, rifaximina (não disponível no Brasil), mostrou eficácia na indução da remissão na DC de grau moderado. Ao contrário do que foi observado na pediatria, a nutrição enteral exclusiva não mostrou eficácia em um grande estudo randomizado e controlado ${ }^{3}$.

A DC deve ser bem caracterizada quanto a sua localização e seu grau de atividade para que escolhas terapêuticas sejam feitas de forma racional com base nas melhores evidências científicas disponíveis até o presente. Além desses parâmetros, a determinação complementar de fatores prognósticos adversos, quando identificados, pode auxiliar nas decisões clínicas, orientando algumas vezes a prescrição de tratamentos de maior eficácia (imunossupressores e biológicos) de maneira mais precoce no sentido de evitar complicações de longo prazo.

\section{REFERÊNCIAS BIBLIOGRÁFICAS}

1. Park JH, Peyrin-Biroulet L, Eisenhut M, Shin JI. Inflammatory bowel diseases (IBD) immunopathogenesis: a comprehensive review of inflammatory molecules. Autoimmunity Reviews. 2017;16:416-26. 
2. Ng SC, Bernstein CN, Vatn MH, Lakatos PL, Loftus EV Jr, Tysk C, et al. Geographical variability and environmental risk factors in inflammatory bowel disease. Gut. 2013;62(4):630-49.

3. Gomollón F, Dignass A, Annese V, Tilg H, Van Assche G, Lindsay JO, et al. $3^{\text {rd }}$ European Evidence-based Consensus on the Diagnosis and Management of Crohn's Disease 2016: Part 1: Diagnosis and medical management ECCO. J Crohns Colitis. 2017;11(1):3-25.

4. Lichtenstein GR, Hanauer SB, Sandborn WJ. Management of Crohn's disease in adults. Practice Parameters Committee of American College of Gastroenterology. Am J Gastroenterol. 2009;104(2):465-83.

5. Satsangi J, Silverberg MS, Vermeire S, Colombel JF. The Montreal classification of inflammatory bowel disease: controversies, consensus, and implications. Gut. 2006;55(6):749-53.

6. Peyrin-Biroulet L, Sandborn W, Sands BE, Reinisch W, Bemelman W, Bryant RV, et al. Selecting therapeutic targets in inflammatory bowel disease (STRIDE): determining therapeutic goals for treat-to-target. Am J Gastroenterol. 2015;110(9):1324-38.

7. Torres J, Caprioli F, Katsanos KH, Lobatón T, Micic D, Zerôncio M, et al. Predicting outcomes to optimize disease management in inflammatory bowel diseases. J Crohns Colitis. 2016;10(12):1385-94.

8. Rezaie A, Kuenzig ME, Benchimol EI, Griffiths AM, Otley AR, Steinhart AH, et al. Budesonide for induction of remission in Crohn's disease. Cochrane Database Syst Rev. 2015;(6):CD000296.

9. Kuenzig ME, Rezaie A, Seow CH, Otley AR, Steinhart AH, Griffiths AM, et al. Budesonide for maintenance of remission in Crohn's disease. Cochrane Database Syst Rev. 2014;(8):CD002913.

10. Hanauer SB, Stromberg U. Oral pentasa in the treatment of active Crohn's disease: a meta-analysis of double-blind, placebo-controlled trials. Clin Gastroenterol Hepatol. 2004;2:379-88.

11. Summers RW, Switz DM, Sessions JT Jr, Becktel JM, Best WR, Kern F Jr, et al. National Cooperative Crohn's Disease Study: results of drug treatment. Gastroenterology. 1979;77(4 Pt 2):847-69.

12. Talley NJ, Abreu MT, Achkar JP, Bernstein CN, Dubinsky MC, Hanauer SB, et al.; American College of Gastroenterology IBD Task Force. An evidence-based systematic review on medical therapies for inflammatory bowel disease. Am J Gastroenterol. 2011;106(Suppl 1):S2-25. 